\title{
Surgery of Labially Impacted Canine \& Orthodontic Management - A Case Report
}

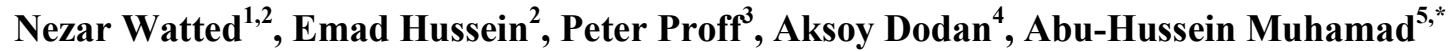 \\ ${ }^{1}$ University Hospital of Würzburg, Clinics and Policlinics for Dental, Oral and Maxillofacial Diseases of the Bavarian \\ Julius-Maximilian-University Wuerzburg, Germany \\ ${ }^{2}$ Department of Orthodontics, Arab American University, Palestine \\ ${ }^{3}$ Department of Orthodontics, University of Regensburg, Germany \\ ${ }^{4}$ Faculty of Dentistry, Department of Orthodontics, Süleyman Demirel University, Turkey \\ ${ }^{5}$ Department of Pediatric Dentistry, University of Athens, Greece
}

Copyright $\bigcirc 2017$ by authors, all rights reserved. Authors agree that this article remains permanently open access under the terms of the Creative Commons Attribution License 4.0 International License

\begin{abstract}
Maxillary canines are one of the most common teeth that are impacted among patients seeking orthodontic treatment. Depending on the position of these impacted teeth, various surgical techniques have been employed for their exposure. His primary goal of surgical phase is to provide the means for correct position of orthodontic anchorage. Additionally, the technique used must ensure favorable tissue anatomy that will permit long-term maintenance of periodontal health. In the present case, a labially impacted maxillary left canine was surgically exposed using an apically positioned flap. Orthodontic extrusion was carried out further.
\end{abstract}

Keywords Impacted Maxillary Canines, Apically Positioned Flap, Attached Gingiva, Mucogingival Interceptive Surgery

\section{Introduction}

The orthodontic treatment of impacted maxillary canine remains a challenge to today's clinicians. The treatment of this clinical entity usually involves surgical exposure of the impacted tooth, followed by orthodontic traction to guide and align it into the dental arch. Bone loss, root resorption, and gingival recession around the treated teeth are some of the most common complications. [1]

Early diagnosis and intervention could save the time, expense, and more complex treatment in the permanent dentition. Tooth impaction can be defined as the infraosseous position of the tooth after the expected time of eruption, whereas the anomalous infraosseous position of the canine before the expected time of eruption can be defined as a displacement. Most of the time, palatal displacement of the maxillary canine results in impaction. $[1,2]$
The second most commonly impacted tooth, after the maxillary third molar, is the maxillary canine, with an incidence from $1 \%$ to $2.5 \%$. Often, the maxillary canine is palatally impacted, with a ratio of about $2: 1 .[1,2,3]$

Impaction of the maxillary canine is a condition in which the tooth is embedded in the alveolus and its eruption is prevented. Maxillary canine impaction is a frequently encountered problem in orthodontic practice. Impacted maxillary canine can be labially, centrally or palatally placed (Fig. 1a, b, Fig. 2a, b, Fig. 3a, b). Eighty-five per cent of impacted maxillary permanent cuspids are palatal and $15 \%$ are labial. $[3,4]$
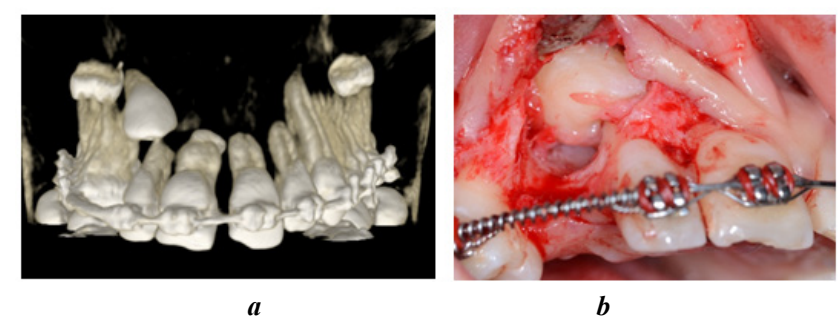

Figure 1a, b. Labially impacted canine 13: Cone Beam Computerized Tomography shows an accurate visualization of what the surgical field will look like, when the exposure is undertaken

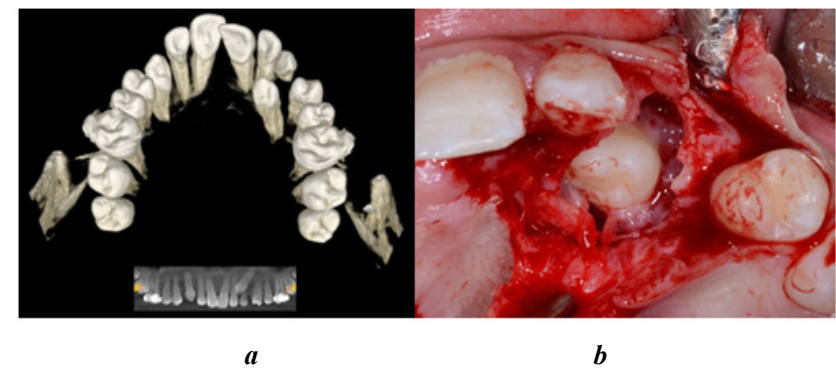

Figure 2a, b. Centrally impacted canine 23 


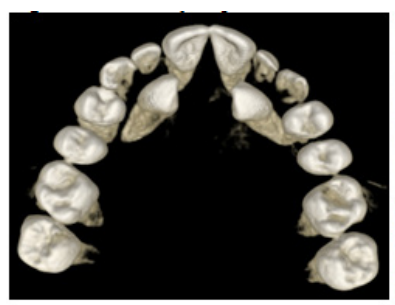

$a$

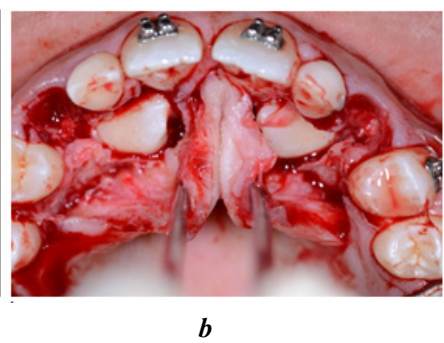

b

Figure 3a, b. Bilateral palatally impacted canine

Several etiologic factors for canine impactions have been proposed: localized, systemic, or genetic. Treatment options for impactions include early diagnosis and interceptive treatment to orthodontic management either by non-extraction or extraction modalities, either by surgical exposure alone or by surgical exposure and attachment with chain -Titanium Button with chain by Watted, Dentaurum, Ispringen/Germany- (Fig. 4a, b) for orthodontic extrusion, forced eruption using micro-implants, surgical removal and autotransplantation.[1, 2, 5]

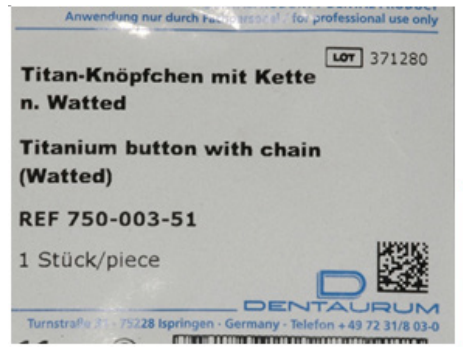

$a$

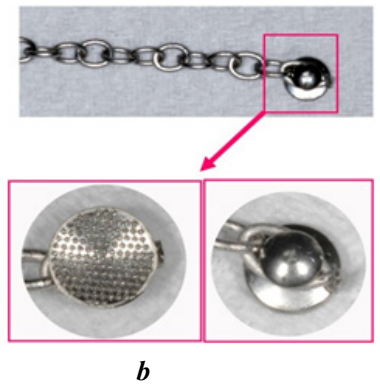

Figure 4a, b. Titanium Button with chain by Watted, Dentaurum, Ispringen/Germany

Causes for ectopic eruption may be either local or general. Generalized causes include systemic diseases such as endocrine deficiencies, febrile diseases, and possibly irradiation. The most common causes for canine impactions are local and can include any of the following factors: (1) tooth size/arch length discrepancies, (2) prolonged retention or early loss of the primary canine, (3) abnormal position of the tooth bud, (4) the presence of an alveolar cleft, (5) ankylosis, (6) cystic or neoplastic formation, (7) dilacerations of the root, (8) iatrogenic origin, and (9) idiopathic condition with no apparent cause. It has also been reported that one cause of maxillary canine impactions may be missing or peg lateral incisors. An increase by 2.4 times in palatally impacted canines adjacent to missing lateral incisors has been reported. This is believed to be attributable to the "guidance theory," that is, that the lateral root serves as a guide along which the canine erupts, and when it is not present or malformed, the canine fails to erupt. $[1,2,3,4,5$, $7,8]$

Location of the impacted tooth can be done clinically and radiographically. In the case of labial impaction, this often can be done by palpation. If the tooth is in the middle of the alveolus or palatally, it would require two intraoral periapical radiographs taken at different angles to determine the location of the tooth. Use of buccal object rule would be useful in determining the location of these impacted teeth [6, 7]. Various surgical techniques have been used to uncover the impacted canine such as, gingivectomy, double pedicle graft, apically positioned flap (APF), free gingival graft, and closed eruption technique. A decision tree to assist in choosing the appropriate technique to correct an impacted canine has been proposed. $[9,10]$

In the present case, a labially impacted maxillary left canine was surgically exposed using a closed eruption technique. Orthodontic extrusion was carried out further.

\section{Case Report}

A 14 year old female patient has presented in the Center for dentistry, research and Aesthetics, Jatt, Israel for orthodontic treatment. On examination maxillary left canine was unerupted, labially displaced, \& was easily palpable on alveolar mucosa apical to the mucogingival junction (Fig. 5a-c). The radiographs obviously showed closed root apices and ectopic eruption of canine whose path of eruption was against the adjacent lateral incisor. The amount of space available between the lateral incisor \& premolar for the canine was not optimal and was blocked due to aberrant eruption direction (Fig. 6 a, b). A gingivectomy procedure could have led to loss of attached gingiva. An apically positioned flap would transform into the attached gingiva on complete eruption of the canine, hence an closed eruption was planned and an informed consent was obtained from the patient.

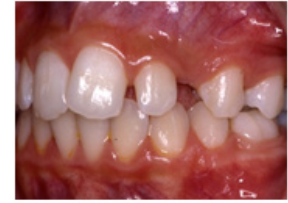

$a$

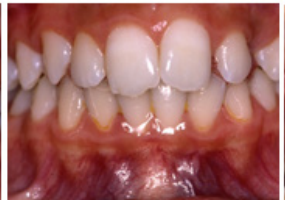

$\boldsymbol{b}$

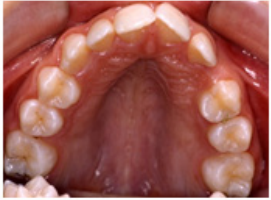

Figure 5a-c. Pre-operative view showing the edentulous site at maxillary left canine

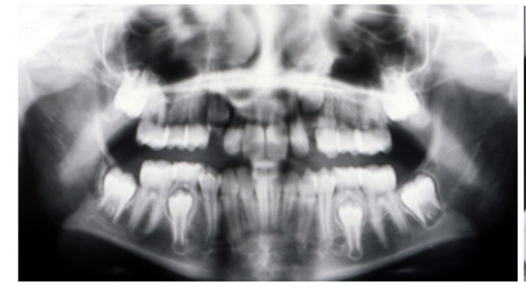

$a$

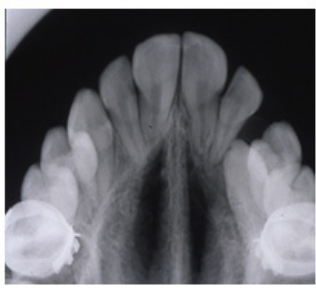

b
Figure 6a, b. The radiographs obviously showed closed root apices

\section{Surgical Procedure}

The surgical area was prepared with adequate anesthesia using 2\% lignocaine $\mathrm{HCl}$ containing 1:80,000 adrenaline 
(Fig. 7a-c).

- Using a No. 15 Bard Parker blade, an initial incision was made on the crest of the edentulous ridge, mesio-distally between the lateral incisor and first premolar teeth (Fig. 7a).

- From the ends of the crestal incision, one vertical releasing incisions were made extending apically beyond the mucogingival junction (Fig. 7b).

- A mucoperiosteal flap was elevated beyond the impacted canine tooth. Fibrous attachment over the canine was removed, exposing the tooth completely (Fig. 7c).

- The mobilized mucoperiosteal flap was moved 1 to the exposed canine tooth (Fig. 7b).

- Orthodontist placed an orthodontic bracket immediately on the exposed canine, under proper isolation.
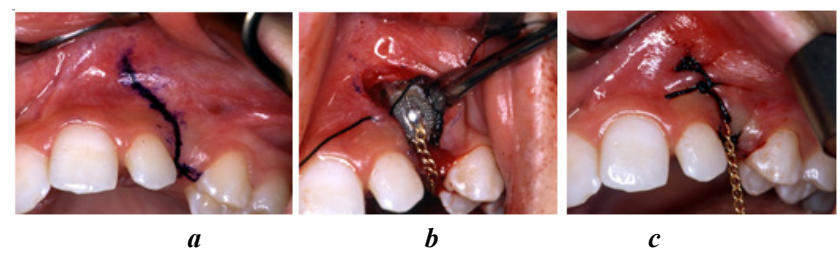

Figure 7a-c. Formation of a Mucoperiosteal flap and expose the crown of an impacted canine with substantial protection of the bone (a), Fixation of the attachment by means of light-curing resin after etching technique (b). repositioned and stitched cloth (closed elongation) (c)

Post-surgical instructions were given and analgesics were prescribed. Patient was recalled after one week for suture removal. At one week follow-up, an uneventful healing was observed and sutures were removed. Orthodontic force was applied immediately using 0.016/0.025 TMA- segment archwire onto the Button with chain placed on surgically exposed canine (Fig. 8 a, b, Fig. 9a, b). At 11 months follow-up visit, the canine was fully in alignment $\&$ showed a healthy keratinized gingiva measuring about $4 \mathrm{~mm}$ width (Fig. 10a, b, Fig. 11a-c).

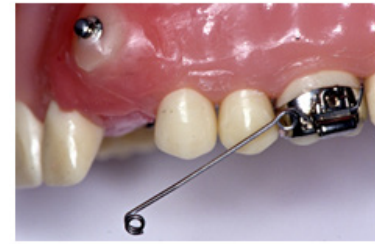

$a$

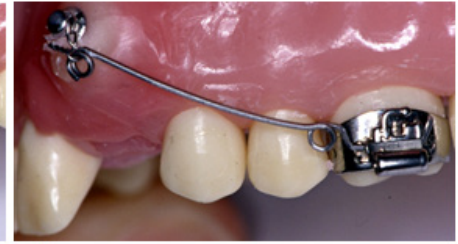

b
Figure 8a, b. Schematic representation of the typodont for TMA segment arcs Passive (a), active (b)

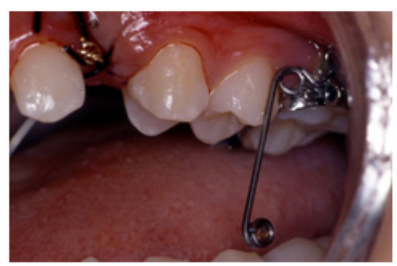

$a$

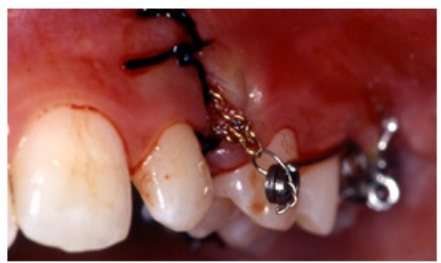

b
Figure 9a, b. Segmental arc passive and active for the extrusion of the canine

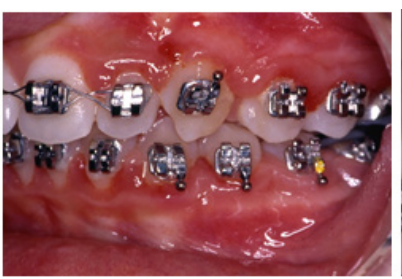

$a$

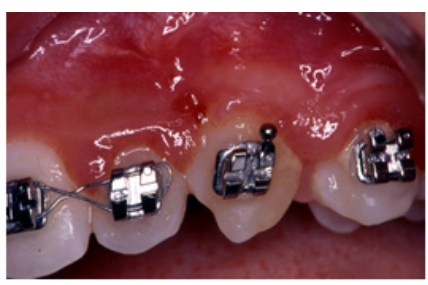

b
Figure 10a, b. 11 months post-operative view showing erupted canine

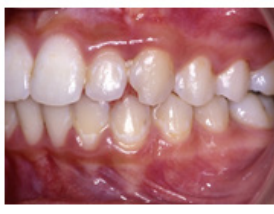

$a$

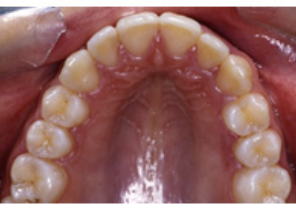

b

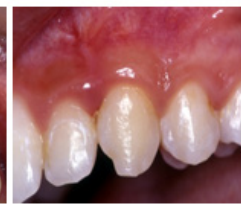

Figure 11a-c. End of the treatment

\section{Discussion}

Maxillary permanent canines are important for an attractive smile and are also essential for a functional occlusion. Therefore, extraction of the labially impacted canine is generally contraindicated. The diagnosis and localization of the impacted teeth is the most important step in the management of impacted teeth. However, bringing an unerupted or impacted maxillary canine into the occlusion should not be the only goal in managing these teeth. The aim should be to attain proper occlusion, a healthy zone of attached gingiva, and ideal alveolar bone height. In the surgical management of the impacted maxillary canine, the choice of flap was either closed-eruption or an apically positioned flap to expose the tooth. Closed-eruption is the surgical method of choice for very high, labially placed maxillary canines. Labially impacted maxillary teeth uncovered with an apically positioned flap technique have more unaesthetic sequelae, such as increased clinical crown length, decreased width of attached tissue, gingival scarring, and intrusive relapse than those uncovered with the closed-eruption technique.[1,2]

Various procedures have been utilized for the surgical exposure of the impacted canines. Gingivectomy could be the treatment of choice if simple excision of tissue would uncover one half or two thirds of the impacted tooth, leaving at least $3 \mathrm{~mm}$ gingival collar apically. [1,7] A double pedicle graft is indicated when the tip of the cusp of the permanent tooth erupts within the zone of keratinized tissue but close to the mucogingival junction[6,7]. If a gingivectomy would not leave enough attached gingiva $\&$ when the cusp of the permanent tooth is erupting apical to the mucogingival junction in the alveolar mucosa it is not possible to move the entrapped gingiva apical to the erupting cusp as a double pedicle flap. This situation makes it prudent to position a flap apically. If the tooth is erupting within the alveolar mucosa distant to the mucogingival junction, an apically placed flap 
is not practical due to the overextended releasing incisions, in such situations a free gingival graft procedure may be selected[7,8,9,10]. These procedures, when selected or performed improperly, result in unfavorable sequelae. In the present case a gingivectomy procedure could have led to loss of attached gingiva \& hence an apically positioned flap was planned. The apically positioned flap had transformed into the attached gingiva on complete eruption of the canine at 3 months post-surgery. This is in accordance with the reports of Pini Prato et al and Vermette et al. It is important, following the flap elevation, that any remaining connective tissue is removed from the labial surface of the tooth $[10,11]$. Further any bone that impedes the eruption of tooth should be removed $\&$ the flap properly adapted. However, no bone was covering the tooth in the present case. It is recommended by many authors that bone is not to be removed from the CEJ as it is this area where the attached gingiva is required to gain attachment. Ideally, the flap should also cover $2-3 \mathrm{~mm}$ of the crown due to which an optimal tissue attachment to radicular $\&$ cemental tissues is achieved. Further, junctional epithelial seals are protected $\&$ even in those cases where teeth move long distances the ability for some marginal migration is retained \& continuing protection of underlying bone is achieved.[11,12,13] A longitudinal study by Pini Prato et al conducted over 7 years, showed that all the three techniques (double pedicle graft, apically positioned flap and free gingival grafts) employed by them appeared to be effective in saving the keratinized tissue for the permanent tooth. In another 2 year longitudinal study Pini Prato et al compared the width of keratinized gingiva after orthodontic therapy for buccally erupting premolars that were pre-treated by extraction of deciduous teeth alone versus mucogingival interceptive surgery $[11,12]$. They concluded that the mucogingival interceptive surgery was an effective technique to maintain keratinized tissue in correspondence with buccally erupting teeth when compared with untreated sites, which had shown significant loss of gingival tissue. In the present case, at the end of 8months following surgical procedure, an esthetically pleasing and a functionally stable band of keratinized gingiva was observed along the labial aspect of canine that was brought into line of occlusion orthodontically.

The amount of space in the dental arch for an unerupted canine can be assessed by performing a space analysis with a full set of orthodontic records. If sufficient space is required in the arch then it can be gained by expansion of the maxillary arch, proclination of maxillary incisors and extraction of the permanent premolars. A surgical exposure of the impacted tooth is indicated when the tooth does not erupt spontaneously after creating enough space in the arch and should be attempted six months after the completion of root formation .[1,7]

Milberg reported a case of a patient with bilateral labial impaction of maxillary canines causing pressure resorption on the lateral aspects of the maxillary central incisors. [14]
Their orthodontic treatment plan included extraction of the impacted canines, positioned between the central and the lateral incisors. Sachan and Chaturvedi reported two cases of extraction treatment approach for buccally displaced or ectopic canine in a patient with severe crowding in the mandibular arch. They concluded that malocclusion with severe crowding is difficult to treat without extraction. [15]

Non-extraction treatment of ectopic canines can compromise the patient's profile and can cause internal or external root resorption of teeth adjacent to the ectopic canine. Similarly in this case report adequate results were achieved through an extraction treatment approach. If the four premolars were not extracted, it might have resulted in proclination of the maxillary and mandibular anterior teeth with a harmful result to the patient's soft tissue profile. By extraction treatment approach, it is much easy to correct the crowding and achieve more stable results.

Gracco et al treated a 14-year-old female patient with buccally impacted upper right permanent canine and supernumerary tooth impeding canine eruption with extraction of supernumerary tooth, surgical exposure of canine with closed eruption technique and orthodontic wire traction. However in the present case it was planned to extract all first premolars followed by surgically exposure of the canine with closed eruption technique and orthodontic wire traction. But canine started to erupt spontaneously after extraction of premolars and surgical exposure of canine was not done. $[16,17]$

Orthodontic treatment is associated with soft and hard tissue changes, thus creation and maintenance of gingival and periodontal health is paramount to ensure optimal results. Although no specific dimensions of keratinized tissue have been indicated for maintenance of periodontal health, in orthodontic cases presence of keratinized tissue is important to prevent formation of periodontal defects $[1,5]$. Classically, three fundamental principles are considered when treating impacted teeth: the surgical approach, the type of fixation that is adhered to the tooth for its posterior traction and the orthodontic movements that have to be applied in order to position the tooth in the dental arch $[11,18,19]$.From a periodontal perspective, the appropriate surgical technique should allow the orthodontist to apply measured forces in a favorable direction for efficient correction of the impaction and for avoidance of damage to adjoining soft tissues and teeth. $[1,5,7,11,20]$

Orthodontic correction of a malocclusion with an impacted maxillary canine takes longer than a similar malocclusion in which all the teeth are erupted. Among the two surgical techniques studied, it was observed that the time required by closed technique was more as compared to open technique, this is in contrast with a study by Chaushu et al., and Pearson et al., which concluded that time required by closed technique was much lesser than open technique. In the present study MIP was used, thereby reducing the time required for bonding an attachment. [21, 22]

Distal surface of the erupted canine showed increase in 
periodontal pocket depth. This finding is in agreement with the findings of study conducted by Wisth et al., and Chaushu et al. The pain perception immediately after the two surgical techniques was similar. However, the postoperative recovery time was much less with closed eruption technique. [23, 24]

The orthodontic guidance of impacted canine may not be successful in all the cases; there may be failures, nonalignment of impacted canine in the desired position. The prognosis is worst in cases where canine is impacted horizontally and apically deep in palatal process. [25] The other factors known for failure include ankylosis of impacted canine, malformations of root, external resorption. Root resorption is reported to be more frequently in palatally impacted canine. An unexplained pain can be because of collision of crown of impacted canine with roots of adjacent teeth.If the impacted canine does not respond to the applied orthodontic force for 3 months, supplementary treatment plan. [26]

\section{Conclusions}

The management of impacted canine is a complex procedure, require a multidisciplinary treatment approach. The clinician should communicate with each other to provide the patient with proper diagnosis, idea of prognosis, and optimal treatment plan based on scientific rationale.

The localization, selection of appropriate surgical approach, conservative management of the soft tissues, rigid anchorage unit, and the direction of the orthodontic traction are the important factors for the successful management of impacted canines. A multidisciplinary approach is therefore essential for treating impacted teeth in order to achieve optimal esthetic and functional outcomes. This case report shows that the judicious selection of the appropriate surgical technique resulted in the formation of an esthetically pleasing and functionally stable band of keratinized gingiva around the canine following its eruption. Coordination between periodontist and orthodontist is crucial in establishing healthy periodontal architecture which could be easily maintained by the patient in the future.

\section{REFERENCES}

[1] Péter Borbély, Nezar Watted, Ivana Dubovská, Viktória Hegedüs ,Abu-Hussein Muhamad; INTERDISCIPLINARY APPROACH IN THE TREATMENT OF IMPACTED CANINES, INTERNATIONAL JOURNAL OF MAXILLOFACIALRESEARCH2015,1,2,116-137

[2] Nezar Watted, Emad Hussein, Obaida Awadi, and Muhamad Abu-Hussein. Transmigration of Impacted Canines: A Report of Two Cases and a Review of the Literature. RRJDS | Volume 2 | Issue 4 | October - December, 2014 ,23-32

[3] Muhamad Abu-Hussein, Nezar Watted, Abdulgani Azzaldeen,
Mohammad Yehia, Obaida Awadi, Yosef Abu-Hussein. Prevalence of Malocclusion and Impacted Canine in Arab Israelian Population (Arab48). International Journal of Public Health Research. Vol. 3, No. 5, 2015, 180-191.

[4] Nezar Watted, Muhamad Abu-Hussein. Prevalence of Impacted Canines in Arab Population in Israel. International Journal of Public Health Research. Vol. 2, No. 6, 2014, pp. 71-77.

[5] Abu-Hussein M, Watted N, Abdulgani M, Abdulgani Az (2016) Tooth Autotransplantation; Clinical Concepts. Journal of Dental and Medical Sciences 15: 105-113

[6] Abu-Hussein Muhamad, and Watted Nezar Miniscrews: Clinical Application of Orthodontic. RRJDS | Volume 2 | Issue 3 | $2014,32-43$

[7] Nezar Watted, Muhamad Abu-Hussein, Obaida Awadi, Borbély Péter; Titanium Button With Chain by Watted For Orthodontic Traction of Impacted Maxillary Canines ,Journal of Dental and Medical Science .Volume 14, Issue 2 Ver. VII (Feb. 2015), 116-

[8] Muhamad Abu-Hussein ,Nezar Watted ,Dana Feştila, Péter Borbély; Surgical-Orthodontic Treatment of Impacted Canines ,Journal of Dental and Medical Sciences, .Volume 14, Issue 10 Ver. $\mathrm{V}$ (Oct. 2015), PP 97-104 DOI: 10.9790/0853-141059710427

[9] Bishara SE. Impacted maxillary canine: A review. Am J Orthod Dentofac Orthop 1992;101:159-71

[10] Kokich VG \& Mathews DP: Surgical \& orthodontic management of impacted teeth. Dent Clin of N Amer 1993; 37 (2): 181-204.

[11] Prato GP, Baccetti T, Magnani C, Agudio G \& Cortellini P: Mucogingival interceptive surgery of buccally-erupted premolars in patients scheduled for orthodontic treatment. I. A 7- year longitudinal study. J periodontol 2000; 71:172-181.

[12] Prato GP, Clauser C \& Cortellini PP: Periodontal plastic and mucogingival surgery. Periodontol 2000 1995; 9: 90-105.

[13] Vermette ME, Kokich VG \& Kennedy DB: Uncovering labially impacted teeth: apically positioned flap \& closed eruption techniques. Angle Orthod. 1994; 5(1):23-34.

[14] Milberg DJ. Labially impacted maxillary canines causing severe root resorption of maxillary central incisors. Angle Orthod. 2006; 76: 173-6.

[15] Sachan A, Chaturvedi T P. Orthodontic management of buccally erupted ectopic canine with two case reports. Contemp Clin Dent 2012; 3:123-8.

[16] Gracco A, Maltoni I, Maltoni M, Zoli L. Eruption of a labially impacted canine using a closed-flap technique and orthodontic wire traction. J Clin Orthod. 2012; 46: 625-30.

[17] Abu-Hussein Muhamad, Watted Nezar, Abdulgani Azzaldeen. The Curve of Dental Arch in Normal Occlusion. Open Science Journal of Clinical Medicine. Vol. 3, No. 2, 2015, 47-54.

[18] Abu-Hussein M, Watted N, Emodi O, Obaida Awadi (2015) management of lower second premolar impaction. Journal Of Dental College Azamgarh 1: 71-79.

[19] Watted N, Abu-Hussein M (2016) Dental Transposition of 
Mandibular Canine and Lateral Incisor. J Dent Probl Solut 3(1): 045-049. DOI: 10.17352/2394-8418.000034

[20] Johnston WD. Treatment of palatally impacted canine teeth. Am J Orthod 1969; 56:589-96.

[21] Chaushu S, Becker A, Zeltser R, Branski S, Vasker N, Chaushu G. patients' perception of recovery after exposure of impacted teeth: A Comparison of closed-versus open-eruption techniques. J Oral Maxillofac Surg 2005; 63:323-9.

[22] Pearson MH, Robinson SN, Reed RT, Birnie DJ, Zaki GA. Management of palatally impacted canines: The findings of a cllaborative study. Eur J Orthod 1997;19:511-5
[23] Wisth PJ, Norderval K, Boe OE. Periodontal status of orthodontically treated impacted maxillary canines. Angle Orthod. 1976; 46:69-76.

[24] Chaushu S, Dykstein N, Ben-Bassat Y, Becker A. Periodontal status of impacted maxillary incisors uncovered by 2 different surgical techniques. J Oral Maxillofac Surg 2009;67:120-4

[25] McSherry PF. The assessment of and treatment options for the buried maxillary canine. Dent Update 1996; 23: 7-10.

[26] Ericson S, Kurol J. Radiographic examination of ectopically erupting maxillary canines. Am J Orthod Dentofac Orthop 1987; 91: 483-92. 